\title{
QUALIDADE DE AMEIXAS 'LAETITIA’ EM FUNÇÃO DA TEMPERATURA E DA ATMOSFERA DE ARMAZENAMENTO ${ }^{1}$
}

\author{
ERLANI DE OLIVEIRA ALVES ${ }^{2}$, CRISTIANO ANDRÉ STEFFENS ${ }^{3}$, \\ CASSANDRO VIDAL TALAMINI DO AMARANTE ${ }^{4}$, AURI BRACKMANN ${ }^{5}$
}

RESUMO - O objetivo deste trabalho foi avaliar o efeito da temperatura e de atmosferas de armazenamento sobre a manutenção da qualidade de ameixas 'Laetitia'. Os tratamentos avaliados constituíram-se na combinação de duas temperaturas $\left(-0,5^{\circ} \mathrm{C}\right.$ e $\left.0,5^{\circ} \mathrm{C}\right)$, com três atmosferas de armazenamento: armazenamento refrigerado (AR), com 21,0 $\mathrm{kPa}$ de $\mathrm{O}_{2}+0,03 \mathrm{kPa}$ de $\mathrm{CO}_{2}$; atmosfera controlada (AC), com 1,0 $\mathrm{kPa}$ de $\mathrm{O}_{2}+$ $3,0 \mathrm{kPa}$ de $\mathrm{CO}_{2}$; e AC, com 2,0 kPa de $\mathrm{O}_{2}+5,0 \mathrm{kPa}$ de $\mathrm{CO}_{2}$. Após 60 dias de armazenamento, foram avaliadas: taxas respiratória e de produção de etileno, acidez titulável (AT), firmeza de polpa, atributos de textura, índice de cor vermelha e ângulo 'hue' $\left(\mathrm{h}^{\circ}\right)$ da casca, e incidência de rachaduras, podridões e degenerescência da polpa. $\mathrm{O}$ armazenamento refrigerado a $-0,5^{\circ} \mathrm{C}$ resultou em menores valores para o índice de cor vermelha, taxa respiratória e de produção de etileno e incidência de frutos rachados. Em ambas as condições de AC, a temperatura de $0,5^{\circ} \mathrm{C}$ resultou em menor índice de cor vermelha, cor da epiderme mais verde, maior firmeza de polpa e menor taxa de produção de etileno, tanto na abertura da câmara como após quatro dias em condição ambiente. As condições de AC retardaram o amadurecimento dos frutos e reduziram a incidência de degenerescência de polpa. $\mathrm{O}$ armazenamento em $\mathrm{AC}$, com $2,0 \mathrm{kPa}$ de $\mathrm{O}_{2}+5,0 \mathrm{kPa}$ de $\mathrm{CO}_{2}$, a $0,5^{\circ} \mathrm{C}$, proporcionou menor taxa respiratória e menor incidência de podridões na saída da câmara, mas maior AT e força para penetração da polpa, após quatro dias em condição ambiente. No entanto, o armazenamento da ameixa 'Laetitia', nas condições de $\mathrm{AC}$ avaliadas, por um período de 60 dias, não reduziu a incidência de degenerescência da polpa.

Termos para indexação: Prunus salicina, pós-colheita, armazenamento, degenerescência de polpa.

\section{QUALITY OF 'LAETITIA' PLUMS WHEN AFFECTED BY TEMPERATURE AND STORAGE ATMOSPHERE}

\begin{abstract}
The aim of this work was to evaluate the effect of temperature and storage atmospheres on the quality of 'Laetitia' plums. The treatments were obtained from the combination of two temperatures $\left(-0.5^{\circ} \mathrm{C}\right.$ and $\left.0.5^{\circ} \mathrm{C}\right)$ with three storage atmospheres: cold storage (CS), with $21.0 \mathrm{kPa}$ of $\mathrm{O}_{2}+0.03 \mathrm{kPa}$ of $\mathrm{CO}_{2}$, and controlled storage atmosphere (CA), with $1.0 \mathrm{kPa}$ de $\mathrm{O}_{2}+3.0 \mathrm{kPa}$ of $\mathrm{CO}_{2}$ and $2.0 \mathrm{kPa}$ of $\mathrm{O}_{2}+5.0$ $\mathrm{kPa}$ of $\mathrm{CO}_{2}$. After 60 days of storage, the fruit were assessed in terms of respiration and ethylene production rates, titratable acidity (TA), flesh firmness, texture attributes, red color index (RCI) and hue angle $\left(\mathrm{h}^{\circ}\right)$ of the skin, and incidence of fruit cracking, decay and flesh browning. Under cold storage, fruit stored at $-0.5^{\circ} \mathrm{C}$ showed lower values of RCI, respiration and ethylene production rates, and incidence of fruit cracking. Under both CA conditions, fruit stored at $0.5^{\circ} \mathrm{C}$ showed lower RCI, greener skin color, higher flesh firmness, and lower ethylene production rate, assessed at removal from cold storage as well as after four days at ambient temperature. The CA conditions delayed fruits ripening and reduced the incidence of flesh browning. Fruit stored under $\mathrm{CA}$, with $2.0 \mathrm{kPa}$ of $\mathrm{O}_{2}+5.0 \mathrm{kPa}$ of $\mathrm{CO}_{2}$, at $0.5^{\circ} \mathrm{C}$, exhibit the lowest respiration rates and incidence of decay assessed at removal from storage, but highest TA and pulp penetration force assessed after shelf life. However, the storage of 'Laetitia' plums for 60 days, under the CA conditions investigated, did not reduce the incidence of flesh browning.
\end{abstract}

Index terms: Prunus salicina, postharvest, storage, flesh browning.

\footnotetext{
'(Trabalho 162-09). Recebido em: 30-06-2009. Aceito para publicação em : 28-07-2010.

${ }^{2}$ M.Sc., Centro de Ciências Agroveterinárias (CAV), Universidade do Estado de Santa Catarina (UDESC), Av. Luiz de Camões, 2090, Caixa Postal 281, CEP 88520-000, Lages-SC. E-mail: erlanea@gmail.com

${ }^{3}$ Dr., Prof. do Departamento de Agronomia, CAV/UDESC, Lages-SC. Autor para correspondência. Email: steffens@cav.udesc.br; ${ }^{4}$ Ph.D., Bolsista de Produtividade em Pesquisa do CNPq, Professor do Departamento de Agronomia, CAV/UDESC, Lages-SC. Email: amarante@cav.udesc.br

${ }^{5}$ Dr., Bolsista de Produtividade em Pesquisa do CNPq, Professor do Departamento de Fitotecnia, Centro de Ciências Rurais (CCR), Universidade Federal de Santa Maria (UFSM), CEP 99105-900. Santa Maria-RS. E-mail: brackman@ccr.ufsm.br
} 


\section{INTRODUÇÃO}

A ameixa 'Laetitia' apresenta maturação tardia, sendo atualmente a cultivar mais utilizada para a implantação de novos pomares de ameixa na região Sul do Brasil. O período de colheita de ameixas normalmente não ultrapassa 20 dias, e o amadurecimento é extremamente rápido, ocorrendo uma grande oferta de frutos em um curto espaço de tempo. Sendo assim, seu armazenamento por um período de 60 dias permitiria a oferta do produto por mais tempo, com melhor remuneração para o produtor. Pode-se prolongar o período de oferta através do armazenamento refrigerado (AR), principal método utilizado para conservação de frutos, pois diminui o metabolismo e evita sua rápida deterioração (BRACKMANN et al., 2003). Apesar de não existir uma recomendação sobre a melhor condição para o armazenamento da ameixa 'Laetitia', sabe-se que o AR prolongado resulta em frutos com baixa firmeza e elevada incidência de degenerescência da polpa, o que pode reduzir a aceitabilidade pelo consumidor (MANGANARIS et al., 2008). Assim, a associação da baixa temperatura com atmosfera controlada (AC) pode constituir uma alternativa para a melhor manutenção da qualidade dos frutos, principalmente em armazenamento prolongado.

Algumas cultivares de ameixa, nectarina e pêssego apresentam melhor manutenção da qualidade quando armazenadas em condição de $\mathrm{AC}$, devido à maior retenção da firmeza de polpa e de ácidos e açúcares, e menor incidência de distúrbios fisiológicos (STREIF, 1995; ZHOU et al., 2000; BRACKMANN et al., 2001; BRACKMANN et al., 2003; DRAKE; ELFVING, 2003; 2004; GIRARDI et al., 2005; STEFFENS et al., 2006, BRACKMANN et al., 2007; MURRAY et al., 2007; SESTARI et al., 2008). Brackmann et al. (2001) verificaram maior firmeza de polpa em ameixas 'Reubennel' armazenadas em AC. Segundo Streif (1995), as melhores condições de AC para ameixas são obtidas com pressões parciais de 1-3kPa para o $\mathrm{O}_{2}$ e de 8-12kPa para o $\mathrm{CO}_{2}$; contudo, devido à sensibilidade da 'Laetitia' à degenerescência da polpa (ARGENTA et al., 2003), condições de alto $\mathrm{CO}_{2}(>5 \mathrm{kPa})$ podem ser inadequadas. Apesar dos benefícios da AC, o seu efeito em ameixas ainda é pouco pesquisado, sendo que para a cultivar Laetitia não existem informações sobre esse sistema de armazenamento.

O objetivo deste trabalho foi avaliar o efeito de duas temperaturas e de três atmosferas de armazenamento sobre a manutenção da qualidade de ameixas 'Laetitia'.

\section{MATERIAL E MÉTODOS}

As ameixas 'Laetitia' foram colhidas em pomar comercial localizado no município de LagesSC, e transportadas para o Laboratório do Núcleo de Pesquisa e Pós-Colheita - NPP, da UFSM em Santa Maria-RS. O transporte rodoviário $(545 \mathrm{~km})$, com duração de aproximadamente $7 \mathrm{~h} 30$, foi realizado com veículo tipo Van, modelo Master, da marca Renault, com os frutos acondicionados em caixas plásticas com $12 \mathrm{~kg}$ de frutos por caixa, para evitar danos por compressão. No laboratório, os frutos foram selecionados, eliminando-se aqueles com lesões, defeitos, ferimentos ou dano mecânico e, posteriormente, procedeu-se à homogeneização das unidades experimentais.

O delineamento experimental utilizado foi o inteiramente casualizado, usando esquema bifatorial $(2 \times 3)$, sendo avaliadas duas temperaturas de armazenamento $\left(-0,5^{\circ} \mathrm{C}\right.$ e $\left.0,5^{\circ} \mathrm{C}\right)$ e três atmosferas de armazenamento: AR, 21,0 $\mathrm{kPa}$ de $\mathrm{O}_{2}+0,03 \mathrm{kPa}$ de $\mathrm{CO}_{2} ; \mathrm{AC1}(1+3)$, com $1,0 \mathrm{kPa}$ de $\mathrm{O}_{2}+3,0 \mathrm{kPa}$ de $\mathrm{CO}_{2}$; e AC2 (2+5) com $2,0 \mathrm{kPa}$ de $\mathrm{O}_{2}+5,0 \mathrm{kPa}$ de $\mathrm{CO}_{2}$. Todos os tratamentos foram mantidos com 95-97\% de UR. Cada tratamento foi constituído por cinco repetições, com 30 frutos por parcela.

Os frutos de todos os tratamentos foram armazenados em minicâmaras experimentais com volume de 180L. As pressões parciais dos gases nos tratamentos com baixo $\mathrm{O}_{2}$ e alto $\mathrm{CO}_{2}$ foram obtidas mediante a diluição do $\mathrm{O}_{2}$ no ambiente de armazenamento com injeção de $\mathrm{N}_{2}$, proveniente de um gerador de nitrogênio, que utiliza o princípio "Pressure Swing Adsorption" (PSA), e posterior injeção de $\mathrm{CO}_{2}$, proveniente de cilindros de alta pressão, até atingir o nível preestabelecido no tratamento. A manutenção das pressões parciais desejadas dos gases, nas diferentes condições de armazenamento, que variavam em função da respiração dos frutos, foi realizada diariamente, com o uso de equipamento automático para controle de gases, da marca Kronenberger/Climasul. Quando os níveis do $\mathrm{CO}_{2}$ e $\mathrm{O}_{2}$ não estavam adequados, o equipamento procedia à correção das pressões parciais até os níveis preestabelecidos. $\mathrm{O} \mathrm{O}_{2}$ consumido pela respiração foi reposto por meio da injeção de ar atmosférico nas minicâmaras, e o $\mathrm{CO}_{2}$ em excesso foi absorvido por uma solução de hidróxido de potássio (40\%), através da qual foi circulado $\mathrm{o}$ ar do ambiente de armazenamento.

Antes do armazenamento, efetuou-se uma análise inicial de duas amostras de 30 frutos para determinar as características da qualidade inicial das ameixas, as quais apresentavam firmeza de polpa 
de $42 \mathrm{~N}$, sólidos solúveis (SS) de 9, $3^{\circ}$ Brix e acidez titulável (AT) de 31,17 meq de ácido cítrico $100 \mathrm{~mL}^{-1}$. Após 60 dias de armazenamento, os frutos foram transportados para o Laboratório de Pesquisa em Fisiologia e Tecnologia Pós-Colheita, da UDESC, em Lages-SC. O transporte dos frutos foi realizado da mesma maneira daquele realizado no momento da colheita. As análises foram realizadas na saída da câmara e após quatro dias de exposição dos frutos à condição de $20 \pm 2^{\circ} \mathrm{C} / 60 \pm 5 \%$ de UR. A análise da saída da câmara foi realizada doze horas após o final do armazenamento, quando os frutos atingiram a temperatura ambiente. As variáveis analisadas foram taxas respiratória e de produção de etileno, acidez titulável (AT), firmeza de polpa, atributos de textura, índice de cor vermelha (ICV), cor da epiderme e incidência de rachaduras, podridões e degenerescência da polpa.

As taxas respiratória e de produção de etileno foram quantificadas, colocando-se 15 frutos de cada amostra em um recipiente de plástico, com o volume de $2.300 \mathrm{~mL}$, que permite fechamento hermético. A taxa respiratória foi obtida pela diferença da concentração de $\mathrm{CO}_{2}$ no interior do recipiente, imediatamente após o seu fechamento e depois de uma hora. Alíquotas de gás $(1 \mathrm{~mL})$ foram retiradas dos recipientes através de um septo e injetadas em um cromatógrafo a gás Varian ${ }^{\circledR}$, modelo CP-3800, equipado com uma coluna Porapak $\mathrm{N}^{\circledR}$ de $3 \mathrm{~m}$ de comprimento (80-100 mesh), metanador e detector de ionização de chama. As temperaturas da coluna, do detector, do metanador e do injetor foram de 45; $120 ; 300$ e $110{ }^{\circ} \mathrm{C}$, respectivamente. Os fluxos de nitrogênio, hidrogênio e ar sintético foram de 70; 30 e $300 \mathrm{~mL} \mathrm{~min}^{-1}$, respectivamente. Os valores da atividade respiratória e da taxa de produção de etileno foram expressos em $\eta$ mol de $\mathrm{CO}_{2} \mathrm{~kg}^{-1} \mathrm{~s}^{-1} \mathrm{e}$ pmol de $\mathrm{C}_{2} \mathrm{H}_{4} \mathrm{~kg}^{-1} \mathrm{~S}^{-1}$, respectivamente. A temperatura dos frutos, no momento das análises de taxas respiratória e de produção de etileno, era de $20 \pm 2^{\circ} \mathrm{C}$.

Os valores de AT (mEq de ácido cítrico $100 \mathrm{~mL}^{-1}$ ) foram obtidos através de uma amostra de $10 \mathrm{~mL}$ de suco, extraída de fatias transversais retiradas da região equatorial dos frutos, em uma centrífuga. Esta amostra foi diluída em $90 \mathrm{~mL}$ de água destilada e titulada com solução de $\mathrm{NaOH}$ a $0,1 \mathrm{M}$ até $\mathrm{pH} 8,1$.

A firmeza de polpa $(\mathrm{N})$ foi determinada na região equatorial dos frutos, em dois lados opostos, após remoção de uma pequena porção da epiderme, com auxílio de um penetrômetro equipado com ponteira de $8 \mathrm{~mm}$ de diâmetro.

Os atributos de textura $(\mathrm{N})$ foram analisados com um texturômetro eletrônico TAXT-plus ${ }^{\circledR}$ (Stable Micro Systems Ltd., Reino Unido), em termos de força necessária para o rompimento da epiderme, para a penetração na polpa e para a compressão do fruto. Para a quantificação da força necessária para o rompimento da epiderme e para a penetração na polpa, foi utilizada ponteira modelo PS2, com $2 \mathrm{~mm}$ de diâmetro, sem a remoção da epiderme, a qual foi introduzida na polpa, a uma profundidade de $5 \mathrm{~mm}$, com velocidades pré-teste, teste e pós-teste de $30 ; 5$ e $30 \mathrm{~mm} \mathrm{~s}^{-1}$, respectivamente. A força necessária para a compressão dos fruto foi determinada usando-se uma plataforma plana, modelo $\mathrm{P} / 75$, com $75 \mathrm{~mm}$ de diâmetro, aplicando-se compressão até uma deformação de $5 \mathrm{~mm}$ na superfície do fruto.

O índice de cor vermelha (ICV) foi determinado avaliando-se a área dos frutos recoberta com coloração vermelha, sendo atribuídas notas de 1 a 4 $(0-25 \%, 26-50 \%, 51-75 \%$ e $76-100 \%$ da superfície do fruto pigmentada de vermelho para os índices 1 ; 2; 3 e 4, respectivamente). O índice foi calculado pelo somatório dos produtos do número de frutos pela sua respectiva nota, dividido pelo total de frutos da amostra.

A determinação da cor da epiderme (ângulo 'hue'; $h^{\circ}$ ) foi efetuada com um colorímetro Minolta, modelo CR 400. As determinações foram realizadas na região equatorial, nos lados menos e mais vermelho dos frutos. $\mathrm{O} \mathrm{h}^{\circ}$ define a coloração básica, sendo que $0^{\circ}=$ vermelho, $90^{\circ}=$ amarelo e $180^{\circ}=$ verde .

A incidência de rachaduras foi realizada pela contagem de frutos que apresentavam rachaduras na epiderme, e os resultados, expressos em percentagem $(\%)$.

A incidência de podridões foi avaliada pela contagem dos frutos afetados e que apresentaram lesões maiores do que $5 \mathrm{~mm}$ de diâmetro e com características de ataque de patógenos. Os resultados foram expressos em percentagem (\%).

A incidência de degenerescência da polpa foi avaliada através de corte transversal na região equatorial do fruto, sendo contabilizados os frutos que apresentaram escurecimento da polpa, sendo os resultados expressos em percentagem (\%).

Os dados foram submetidos à análise da variância (ANOVA), sendo que aqueles obtidos em percentagem foram transformados pela fórmula arco-seno $[(\mathrm{x}+0,5) / 100]^{1 / 2}$ antes de serem analisados. Para a comparação das médias, adotou-se o teste de Tukey $(P<0,05)$. 


\section{RESULTADOS E DISCUSSÃO}

Houve interação significativa entre os fatores avaliados, temperatura e atmosfera de armazenamento, tanto na saída da câmara como após quatro dias de simulação de vida de prateleira, para as variáveis taxas respiratória e de produção de etileno, AT e força para ruptura da casca (Tabelas 1 e 2), e apenas na saída da câmara para as variáveis índice de cor vermelha, $\mathrm{h}^{\circ}$ no lado mais vermelho do fruto e forças para penetração da polpa e compressão do fruto (Tabelas 2 e 3 ).

Após 60 dias de armazenamento, a menor taxa respiratória foi observada em frutos armazenados a $-0,5^{\circ} \mathrm{C}$, independentemente da composição gasosa do armazenamento (Tabela 1). Após quatro dias sob condição ambiente, os frutos mantidos a $-0,5^{\circ} \mathrm{C}$ em $\mathrm{AR}$ apresentaram menor taxa respiratória do que aqueles armazenados em $\mathrm{AR}$ a $0,5^{\circ} \mathrm{C}$ (Tabela 1). A redução da temperatura de armazenamento reduz o metabolismo celular (WILLS et al., 1998) e a taxa respiratória dos frutos (DRAKE; ELFVING, 2003; STEFFENS et al., 2007).

Com relação ao efeito da $\mathrm{AC}$, o armazenamento na condição de $2,0 \mathrm{kPa}$ de $\mathrm{O}_{2}+5,0 \mathrm{kPa}$ de $\mathrm{CO}_{2}$ proporcionou menor taxa respiratória, na saída da câmara, mas após quatro dias em condição ambiente, as duas condições de AC avaliadas reduziram a taxa respiratória dos frutos, em relação à condição de $\mathrm{AR}$, apenas nos armazenados a $0,5^{\circ} \mathrm{C}$ (Tabela 1). Este efeito da $\mathrm{AC}$ deve-se ao baixo $\mathrm{O}_{2}$ e ao alto $\mathrm{CO}_{2}$, e tem sido observado em outros trabalhos (MATHOOKO, 1996; FONSECA et al., 2002; LIU et al., 2004; STEFFENS et al., 2007). De acordo com Kader (1987), a redução na atividade respiratória pelo baixo $\mathrm{O}_{2}$ é decorrente do decréscimo na atividade de várias oxidases, tais como citocromo oxidase, polifenoloxidase, ácido ascórbico oxidase e ácido glicólico oxidase. Quanto ao efeito do $\mathrm{CO}_{2}$ sobre a respiração, algumas hipóteses têm sido formuladas: ele pode reduzir a respiração diretamente, inibindo a rota glicolítica, agindo na fosfofrutoquinase, e o ciclo dos ácidos tricarboxílicos, agindo na succinato oxidase e na isocitrato desidrogenase, e indiretamente, reduzindo a ação do etileno sobre enzimas envolvidas no processo respiratório (MATHOOKO, 1996; FONSECA et al., 2002; LIU et al., 2004). Já o fato de não ter sido observado efeito da atmosfera controlada sobre a taxa respiratória nos frutos armazenados a $-0,5^{\circ} \mathrm{C}$, após quatro dias de exposição dos frutos em condição ambiente, pode estar relacionado ao fato dessa temperatura, por estar próxima ao limite crítico de armazenamento, juntamente com as condições de atmosfera controlada terem causado uma grande redução na atividade respiratória durante o armazenamento, conforme observado na saída da câmara (Tabela 1). Essa intensa redução na atividade respiratória deve ter reduzido o consumo de substratos respiratórios, os quais, após exposição dos frutos a condições normais de temperatura e composição de gases, puderam ser metabolizados, contribuindo para maior taxa respiratória.

A taxa de produção de etileno, na saída da câmara, foi menor nos frutos armazenados a $-0,5^{\circ} \mathrm{C}$, em todas as atmosferas avaliadas (Tabela 1). A menor taxa de produção de etileno em temperaturas mais baixas de armazenamento está relacionada à redução no metabolismo dos frutos (WILLS et al., 1998). Na temperatura de $-0,5^{\circ} \mathrm{C}$, na saída da câmara, não houve diferença entre as atmosferas de armazenamento quanto à taxa de produção de etileno (Tabela 1). Conforme se observa na Tabela 1, a taxa de produção de etileno foi muito baixa nos frutos mantidos nessa temperatura, em todas as atmosferas avaliadas, o que evidencia o forte efeito da temperatura de $-0,5^{\circ} \mathrm{C}$ na redução da produção de etileno, devido à diminuição do metabolismo celular, não apresentando efeito sinérgico com a atmosfera controlada. Contudo, após quatro dias em condição ambiente, na temperatura de $-0,5^{\circ} \mathrm{C}$, foi verificada maior taxa de produção de etileno na condição de $2,0 \mathrm{kPa}$ de $\mathrm{O}_{2}+5,0 \mathrm{kPa}$ de $\mathrm{CO}_{2}$. Essa maior taxa de produção de etileno, nesse tratamento, pode estar relacionada ao estresse causado aos frutos pelo efeito combinado da baixa temperatura $\left(-0,5^{\circ} \mathrm{C}\right)$ com o alto $\mathrm{CO}_{2}(5,0 \mathrm{kPa})$, pois condições extremas de armazenamento induzem a produção de etileno, em decorrência do estresse fisiológico aos tecidos nessas condições de armazenamento (STEFFENS et al., 2006).

Com relação ao armazenamento a $0,5^{\circ} \mathrm{C}$, os frutos em AC apresentaram menor taxa de produção de etileno do que naqueles sob AR, tanto na saída da câmara como após quatro dias em condição ambiente. A menor taxa de produção de etileno em AC, observada nos frutos mantidos a $0,5^{\circ} \mathrm{C}$, deve estar relacionada à menor oxidação do $\mathrm{ACC}$, devido ao baixo $\mathrm{O}_{2}$, e/ou pelo efeito inibitório do $\mathrm{CO}_{2}$ na ação do etileno em induzir sua autocatálise (BLANKENSHIP; DOLE, 2003). Além disso, o elevado $\mathrm{CO}_{2}$ pode reduzir o $\mathrm{pH}$ da célula, podendo assim diminuir a atividade da ACC oxidase e, portanto, a biossíntese do etileno (GORNY; KADER, 1994).

A $0,5^{\circ} \mathrm{C}$, os maiores valores de AT, na saída da câmara, foi observada nos frutos armazenados em $\mathrm{AC}$, independentemente das pressões parciais de $\mathrm{O}_{2} \mathrm{e}$ $\mathrm{CO}_{2}$, sendo que a $-0,5^{\circ} \mathrm{C}$ não houve efeito da atmosfera de armazenamento. Após quatro dias em condição 
ambiente, a AT apresentou os maiores valores na atmosfera de 2,0 $\mathrm{kPa} \mathrm{O}_{2}+5,0 \mathrm{kPa} \mathrm{CO}$, seguida pela atmosfera $1,0 \mathrm{kPa} \mathrm{O}_{2}+3,0 \mathrm{kPa} \mathrm{CO}$, em ambas as temperaturas de armazenamento (Tabela 1). Resultados similares foram reportados por Brackmann et al. (2007) e Sestari et al. (2008). Todavia, Drake e Elfving (2003) encontraram menor AT em condição de AC. Os maiores valores de AT em AC devem-se, em parte, à baixa taxa respiratória nestas condições de armazenamento (Tabela 1), principalmente nos frutos armazenados em 2,0 $\mathrm{kPa} \mathrm{O}_{2}+5,0 \mathrm{kPa} \mathrm{CO}$. É provável que essas pressões parciais de $\mathrm{O}_{2}$ e $\mathrm{CO}_{2}$ tenham reduzido o consumo dos ácidos orgânicos no processo respiratório (STEFFENS et al., 2007; SESTARI et al., 2008).

A temperatura de $0,5^{\circ} \mathrm{C}$ proporcionou frutos com maior firmeza de polpa, independentemente da atmosfera de armazenamento, na saída da câmara. Porém, não houve diferença entre as temperaturas de armazenamento, na avaliação realizada após quatro dias em condição ambiente (Tabela 2). Contudo, os frutos armazenados em AC apresentaram maior firmeza de polpa do que os frutos em AR, em ambas as avaliações (Tabela 2). Resultados semelhantes foram obtidos por outros trabalhos em ameixa (BRACKMANN et al., 2001), nectarina (DRAKE; ELFVING, 2003) e pêssego (SESTARI et al., 2008).

Em AR, a maior força de ruptura da epiderme foi observada na temperatura de $-0,5^{\circ} \mathrm{C}$, enquanto em AC não houve diferença entre as temperaturas, nas duas avaliações (Tabela 2). Considerando as atmosferas de armazenamento, observou-se que as condições de $\mathrm{AC}$ proporcionaram maiores valores de força para ruptura da epiderme. A maior força para a penetração da polpa e para a compressão do fruto, na saída da câmara, foi observada nas condições de $\mathrm{AC}$ combinadas com $0,5^{\circ} \mathrm{C}$. Contudo, após quatro dias de exposição dos frutos em condição ambiente, os maiores valores foram observados nos frutos em $\mathrm{AC}$, independentemente da temperatura de armazenamento (Tabela 2). A força para penetração da polpa, após quatro dias de exposição dos frutos em condição ambiente, apresentou diferenças entre as condições de $\mathrm{AC}$, sendo observados maiores valores a $2,0 \mathrm{kPa}$ de $\mathrm{O}_{2}+5,0 \mathrm{kPa}$ de $\mathrm{CO}_{2}$ (Tabela 2). O efeito da AC sobre a manutenção da firmeza de polpa e dos atributos de textura pode estar relacionado à sua atuação na redução da biossíntese e ação de etileno, reduzindo a atividade das enzimas hidrolíticas responsáveis pela degradação dos componentes da parede celular. Majumder e Mazumdar (2002) constataram que o aumento na atividade da enzima poligalacturonase apresentou correlação positiva com a evolução de etileno.

Os frutos armazenados em AC apresentaram, na saída da câmara a $0,5^{\circ} \mathrm{C}$, menores valores de ICV e maiores valores de $h^{\circ}$ (medidos nos lados mais e menos vermelho dos frutos) na casca (Tabela 3 ). Esses resultados demonstram que os frutos armazenados em AC apresentaram menor percentagem de cor vermelha na epiderme, bem como uma coloração vermelha menos intensa. Assim como na firmeza de polpa e nos atributos de textura, a manutenção da coloração da epiderme nos frutos deve estar relacionada à menor biossíntese e ação do etileno no armazenamento em $\mathrm{AC}$, pois a mudança na cor durante o amadurecimento de ameixas é um processo dependente da ação deste fitormônio (ARGENTA et al., 2003).

Apenas frutos armazenados em AR, na temperatura de $0,5^{\circ} \mathrm{C}$, apresentaram incidência de rachaduras na saída da câmara, correspondente a $20 \%$, sendo nula nos demais tratamentos (dados não apresentados). Em maçãs 'Royal Gala' e 'Galaxy', o baixo $\mathrm{O}_{2}$ e o alto $\mathrm{CO}_{2}$ em condição de $\mathrm{AC}$ reduziram a ocorrência de rachaduras, em relação ao $\mathrm{AR}$, como resultado do retardo no amadurecimento dos frutos (BRACKMANN et al., 2008), corroborando os resultados do presente trabalho.

A menor incidência de podridões, na saída da câmara, foi verificada a $-0,5^{\circ} \mathrm{C}$, em comparação à temperatura de $0,5^{\circ} \mathrm{C}$. Com relação às atmosferas de armazenamento, os frutos armazenados a 2,0 $\mathrm{kPa}$ de $\mathrm{O}_{2}+5,0 \mathrm{kPa}$ de $\mathrm{CO}_{2}$ apresentaram, na saída da câmara, menor incidência de podridão. Porém, após quatro dias em condição ambiente, não foi observado diferença entre as atmosferas e as temperaturas de armazenamento (Tabela 4). A menor incidência de podridões nos frutos armazenados em AC deve-se ao aumento na resistência física dos tecidos dos frutos, evidenciado principalmente pela maior firmeza de polpa, devido ao efeito da condição de AC no retardo do amadurecimento (SAQUET et al., 1997; JAYAS; JEYAMKONDAN, 2002).

$\mathrm{O}$ armazenamento na temperatura de $0,5^{\circ} \mathrm{C}$ reduziu a incidência de degenerescência de polpa em relação à temperatura de $-0,5^{\circ} \mathrm{C}$, tanto na saída da câmara como após quatro dias em condição ambiente (Tabela 4 ). A temperatura de $-0,5^{\circ} \mathrm{C}$ pode ter causado estresse ao fruto e, assim, aumentado a incidência de degenerescência da polpa. Na saída da câmara, não houve diferença quanto à incidência de degenerescência de polpa entre as atmosferas de armazenamento. No entanto, após quatro dias de exposição dos frutos em condição ambiente, os frutos armazenados em AC apresentaram os menores valores de incidência de degenerescência de polpa (Tabela 4). Brackmann et al. (2003) também verificaram maior degenerescência da polpa em pêssegos 
'Chiripá' armazenados em AR do que em AC. Os resultados de degenerescência de polpa evidenciam que a ameixa 'Laetitia' não suporta o armazenamento por 60 dias nas condições avaliadas. Apesar do retardo no amadurecimento dos frutos armazenados nas condições de AC, ainda houve elevada incidência de degenerescência da polpa, comprometendo a qualidade dos frutos e, possivelmente, também a aceitação do produto pelos consumidores. Talvez o armazenamento em condições de $\mathrm{AC}$, com níveis maiores de $\mathrm{O}_{2}$ e/ou mais baixos de $\mathrm{CO}_{2}$, bem como a combinação com outras tecnologias pós-colheita, possa permitir o armazenamento dos frutos durante esse período sem o desenvolvimento de degenerescência da polpa.

TABELA 1 - Taxas respiratória e de produção de etileno e acidez titulável, em ameixas 'Laetitia' armazenadas por 60 dias sob refrigeração $\left(0,5^{\circ} \mathrm{C} \mathrm{e}-0,5^{\circ} \mathrm{C} ; 95-97 \%\right.$ UR) e $\mathrm{AC}$, mais quatro dias em condição ambiente $\left(20 \pm 2^{\circ} \mathrm{C} ; 60 \pm 5 \%\right.$ UR).

\begin{tabular}{|c|c|c|c|c|}
\hline \multirow{3}{*}{ Atmosfera } & \multicolumn{2}{|c|}{ Saída da câmara } & \multicolumn{2}{|c|}{ Após quatro dias em condição ambiente } \\
\hline & $-0,5^{\circ} \mathrm{C}$ & $0,5^{\circ} \mathrm{C}$ & $-0,5^{\circ} \mathrm{C}$ & $0,5^{\circ} \mathrm{C}$ \\
\hline & \multicolumn{4}{|c|}{ Taxa respiratória $\left(\eta \mathrm{mol} \mathrm{CO}_{2} \mathrm{~kg}^{-1} \mathrm{~s}^{-1}\right)$} \\
\hline$\overline{\mathrm{AR}}$ & $219,40 \mathrm{aB}$ & $775,70 \mathrm{aA}$ & $289,00 \mathrm{aB}$ & $498,50 \mathrm{aA}$ \\
\hline $\mathrm{AC} 1$ & $153,90 \mathrm{bB}$ & $757,50 \mathrm{aA}$ & $326,00 \mathrm{aA}$ & $298,90 \mathrm{bA}$ \\
\hline $\mathrm{AC} 2$ & $98,10 \mathrm{cB}$ & $529,60 \mathrm{bA}$ & $291,70 \mathrm{aA}$ & $362,20 \mathrm{bA}$ \\
\hline \multirow[t]{2}{*}{ C.V. $(\%)$} & \multicolumn{2}{|c|}{10,65} & \multicolumn{2}{|c|}{16,66} \\
\hline & \multicolumn{4}{|c|}{ 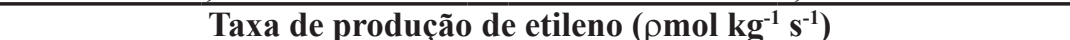 } \\
\hline$\overline{\mathrm{AR}}$ & $0,46 \mathrm{aB}$ & $5,54 \mathrm{aA}$ & $7,89 \mathrm{bB}$ & $15,60 \mathrm{aA}$ \\
\hline $\mathrm{AC} 1$ & $0,72 \mathrm{aB}$ & $4,94 \mathrm{bA}$ & $4,60 \mathrm{bA}$ & $5,14 \mathrm{bA}$ \\
\hline $\mathrm{AC} 2$ & $0,53 \mathrm{aB}$ & $3,47 \mathrm{bA}$ & $17,70 \mathrm{aA}$ & $3,44 \mathrm{bB}$ \\
\hline \multirow[t]{2}{*}{ C.V.(\%) } & & & & \\
\hline & \multicolumn{4}{|c|}{ Acidez titulável ( $\mathbf{m E q}$ de ácido cítrico $\left.100 \mathbf{m L}^{-1}\right)$} \\
\hline$\overline{\mathrm{AR}}$ & $12,80 \mathrm{aA}$ & $12,80 \mathrm{bA}$ & $11,10 \mathrm{cA}$ & $10,40 \mathrm{cA}$ \\
\hline $\mathrm{AC} 1$ & $13,20 \mathrm{aB}$ & $19,40 \mathrm{aA}$ & $13,30 \mathrm{bB}$ & $15,60 \mathrm{bA}$ \\
\hline $\mathrm{AC} 2$ & $14,00 \mathrm{aB}$ & $20,20 \mathrm{aA}$ & $15,00 \mathrm{aB}$ & $19,50 \mathrm{aA}$ \\
\hline$\overline{\mathrm{CV}}(\%)$ & \multicolumn{2}{|c|}{$\overline{7,02}$} & \multicolumn{2}{|c|}{8,47} \\
\hline
\end{tabular}

Médias seguidas por mesma letra, maiúscula na horizontal, entre temperaturas (para uma mesma data de avaliação), e minúscula na vertical, entre atmosferas de armazenamento, não diferem entre si, pelo teste de Tukey $(\mathrm{p}<0,05)$. AR $\left(21 \mathrm{kPa} \mathrm{O}_{2}+<0,03 \mathrm{kPa}^{\mathrm{CO}} \mathrm{O}_{2}\right)$, $\mathrm{AC} 1(1 \mathrm{kPa} \mathrm{O}+3 \mathrm{kPaCO})$ e $\mathrm{AC} 2\left(2 \mathrm{kPaO}_{2}+5 \mathrm{kPaCO}_{2}\right)$ 
TABELA 2 - Firmeza de polpa e força para ruptura da epiderme, penetração da polpa e compressão do fruto, em ameixas 'Laetitia' armazenadas por 60 dias sob refrigeração $\left(0,5^{\circ} \mathrm{C}\right.$ e $-0,5^{\circ} \mathrm{C} ; 95-97 \%$ UR) e AC, mais quatro dias em condição ambiente $\left(20 \pm 2^{\circ} \mathrm{C} ; 60 \pm 5 \% \mathrm{UR}\right)$.

\begin{tabular}{|c|c|c|c|c|c|c|}
\hline \multirow{3}{*}{ Atmosfera } & \multicolumn{3}{|c|}{ Saída da câmara } & \multicolumn{3}{|c|}{ Após quatro dias em condição ambiente } \\
\hline & $-0,5^{\circ} \mathrm{C}$ & $0,5^{\circ} \mathrm{C}$ & Média & $-0,5^{\circ} \mathrm{C}$ & $0,5^{\circ} \mathrm{C}$ & Média \\
\hline & \multicolumn{6}{|c|}{ Firmeza de polpa $(\mathrm{N})$} \\
\hline$\overline{\mathrm{AR}}$ & 22,50 & 16,90 & $19,70 b$ & 19,30 & 12,20 & $15,70 \mathrm{~b}$ \\
\hline $\mathrm{AC} 1$ & 30,50 & 35,60 & $33,10 \mathrm{a}$ & 36,00 & 33,80 & $34,90 \mathrm{a}$ \\
\hline $\mathrm{AC} 2$ & 32,30 & 40,50 & $36,40 \mathrm{a}$ & 25,80 & 35,80 & $30,80 \mathrm{a}$ \\
\hline Média & $28,4 \mathrm{~B}$ & $31,0 \mathrm{~A}$ & - & $27,0 \mathrm{~A}$ & $27,3 \mathrm{~A}$ & - \\
\hline \multirow[t]{2}{*}{$\underline{\mathrm{CV}(\%)}$} & & & & & & \\
\hline & \multicolumn{6}{|c|}{ Força de ruptura da epiderme $(\mathrm{N})$} \\
\hline$\overline{\mathrm{AR}}$ & $6,35 \mathrm{bA}$ & $4,42 \mathrm{bB}$ & - & $5,43 \mathrm{bA}$ & $3,79 \mathrm{bB}$ & - \\
\hline $\mathrm{AC} 1$ & $9,60 \mathrm{aA}$ & $9,30 \mathrm{aA}$ & - & $9,80 \mathrm{aA}$ & $10,10 \mathrm{aA}$ & - \\
\hline $\mathrm{AC} 2$ & $10,10 \mathrm{aA}$ & $9,31 \mathrm{aA}$ & - & $9,47 \mathrm{aA}$ & $9,91 \mathrm{aA}$ & - \\
\hline \multirow{2}{*}{$\mathrm{CV}(\%)$} & \multicolumn{3}{|c|}{6,66} & \multicolumn{3}{|c|}{4,25} \\
\hline & \multicolumn{6}{|c|}{ Força para a penetração da polpa $(\mathbf{N})$} \\
\hline$\overline{\mathrm{AR}}$ & $1,50 \mathrm{aA}$ & $1,11 \mathrm{bA}$ & - & 1,18 & 0,87 & $1,03 \mathrm{c}$ \\
\hline $\mathrm{AC} 1$ & $1,70 \mathrm{aB}$ & $2,56 \mathrm{aA}$ & - & 1,97 & 2,04 & $2,00 \mathrm{~b}$ \\
\hline $\mathrm{AC} 2$ & $1,65 \mathrm{aB}$ & $2,49 \mathrm{aA}$ & - & 2,41 & 2,17 & $2,29 \mathrm{a}$ \\
\hline Média & - & - & - & $1,85 \mathrm{~A}$ & $1,69 \mathrm{~B}$ & \\
\hline \multirow[t]{2}{*}{$\mathrm{CV}(\%)$} & \multicolumn{3}{|c|}{14,17} & \multicolumn{3}{|c|}{10,21} \\
\hline & \multicolumn{6}{|c|}{ Forca para a compressão do fruto $(\mathbf{N})$} \\
\hline$\overline{A R}$ & $57,90 \mathrm{aA}$ & $42,80 \mathrm{bA}$ & - & 43,40 & 32,00 & $37,70 \mathrm{~b}$ \\
\hline $\mathrm{AC} 1$ & $67,60 \mathrm{aA}$ & $78,30 \mathrm{aA}$ & - & 74,10 & 74,40 & $74,20 \mathrm{a}$ \\
\hline $\mathrm{AC} 2$ & $65,20 \mathrm{aB}$ & $101,0 \mathrm{aA}$ & - & 73,80 & 80,40 & $77,10 \mathrm{a}$ \\
\hline Média & \multirow{2}{*}{\multicolumn{2}{|c|}{16,43}} & - & $63,8 \mathrm{~A}$ & $62,3 \mathrm{~A}$ & \\
\hline $\mathrm{CV}(\%)$ & & & & \multicolumn{2}{|c|}{10,7} & \\
\hline
\end{tabular}

Médias seguidas por mesma letra, maiúscula na horizontal, entre temperaturas (para uma mesma data de avaliação), e minúscula na vertical, entre atmosferas de armazenamento, não diferem entre si, pelo teste de Tukey $(\mathrm{p}<0,05)$. $\mathrm{AR}\left(21 \mathrm{kPa} \mathrm{O}_{2}+<0,03 \mathrm{kPa}^{\mathrm{CO}}\right.$ ), $\mathrm{AC} 1\left(1 \mathrm{kPaO}_{2}+3 \mathrm{kPaCO}\right)$ e $\left.\mathrm{AC} 2\left(2 \mathrm{kPaO}_{2}+5 \mathrm{kPa} \mathrm{CO}\right)_{2}\right)$

TABELA 3 - Índice de cor vermelha e ângulo 'hue' $\left(h^{\circ}\right)$ em ameixas 'Laetitia' armazenadas por 60 dias sob refrigeração $\left(0,5^{\circ} \mathrm{C} \mathrm{e}-0,5^{\circ} \mathrm{C} ; 95-97 \% \mathrm{UR}\right)$ e $\mathrm{AC}$, mais quatro dias em condição ambiente $\left(20 \pm 2^{\circ} \mathrm{C} ; 60 \pm 5 \%\right.$ UR).

\begin{tabular}{|c|c|c|c|c|c|c|}
\hline \multirow{3}{*}{ Atmosfera } & \multicolumn{3}{|c|}{ Saída da Câmara } & \multicolumn{3}{|c|}{ Após quatro dias em condição ambiente } \\
\hline & $-0,5^{\circ} \mathrm{C}$ & $0,5^{\circ} \mathrm{C}$ & Média & $-0,5^{\circ} \mathrm{C}$ & $0,5^{\circ} \mathrm{C}$ & Média \\
\hline & \multicolumn{6}{|c|}{ Índice de cor vermelha $(1-4) * * *$} \\
\hline$\overline{\mathrm{AR}}$ & $2,91 \mathrm{aB}$ & $3,15 \mathrm{aA}$ & - & 3,94 & 3,91 & $3,93 a$ \\
\hline $\mathrm{AC} 1$ & $2,78 \mathrm{aA}$ & $2,84 \mathrm{bA}$ & - & 3,18 & 4,01 & $3,66 \mathrm{a}$ \\
\hline $\mathrm{AC} 2$ & $3,02 \mathrm{aA}$ & $2,17 \mathrm{bA}$ & - & 3,58 & 3,49 & $3,53 \mathrm{a}$ \\
\hline Média & - & & - & $3,57 \mathrm{~A}$ & $3,84 \mathrm{~A}$ & - \\
\hline \multirow[t]{2}{*}{$\underline{\mathrm{CV}}(\%)$} & \multicolumn{3}{|c|}{16,43} & \multicolumn{2}{|c|}{16,26} & \\
\hline & \multicolumn{6}{|c|}{$\mathrm{h}^{\mathbf{0}}$ (lado vermelho) } \\
\hline AR & $36,0 \mathrm{aA}$ & $29,0 \mathrm{bA}$ & - & 25,3 & 23,1 & $24,2 b$ \\
\hline $\mathrm{ACl}$ & $41,6 \mathrm{aA}$ & $35,6 \mathrm{aA}$ & - & 36,4 & 30,2 & $33,3 \mathrm{a}$ \\
\hline$\underline{\mathrm{AC} 2}$ & $38,4 \mathrm{aA}$ & $38,2 \mathrm{aA}$ & - & 34,1 & 31,9 & $32,9 \mathrm{a}$ \\
\hline Média & - & - & - & $31,9 \mathrm{~A}$ & $28,4 \mathrm{~B}$ & - \\
\hline \multirow[t]{2}{*}{$\underline{\mathrm{CV}}(\%)$} & \multicolumn{2}{|r|}{5,94} & & \multicolumn{2}{|c|}{8,06} & \\
\hline & \multicolumn{6}{|c|}{$\mathbf{h}^{0}$ (lado verde) } \\
\hline AR & 67,2 & 76,1 & $71,6 b$ & 35,3 & 41,6 & $38,5 b$ \\
\hline $\mathrm{AC} 1$ & 76,9 & 91,2 & $84,1 \mathrm{a}$ & 61,4 & 76,8 & $69,1 \mathrm{a}$ \\
\hline $\mathrm{AC} 2$ & 73,7 & 87,7 & $80,7 \mathrm{a}$ & 62,3 & 68,7 & $65,5 \mathrm{a}$ \\
\hline Média & $72,6 \mathrm{~B}$ & $85,0 \mathrm{~A}$ & - & $53,0 \mathrm{~B}$ & $62,4 \mathrm{~A}$ & - \\
\hline CV (\%) & \multicolumn{3}{|c|}{6,34} & \multicolumn{2}{|c|}{9,83} & \\
\hline
\end{tabular}

Médias seguidas por mesma letra, maiúscula na horizontal, entre temperaturas (para uma mesma data de avaliação), e minúscula na vertical, entre atmosferas de armazenamento, não diferem entre si, pelo teste de Tukey $(\mathrm{p}<0,05)$. $\mathrm{AR}\left(21 \mathrm{kPa} \mathrm{\textrm {O } _ { 2 }}+<0,03 \mathrm{kPa} \mathrm{CO}_{2}\right)$, $\mathrm{AC} 1\left(1 \mathrm{kPa} \mathrm{O}+3 \mathrm{kPaCO}_{2}\right)$ e $\mathrm{AC} 2\left(2 \mathrm{kPaO}_{2}+5 \mathrm{kPa} \mathrm{CO}_{2}\right)$ 
TABELA 4 - Incidência de frutos com podridão e degenerescência da polpa, em ameixas 'Laetitia' armazenadas por 60 dias sob refrigeração $\left(0,5^{\circ} \mathrm{C}\right.$ e $-0,5^{\circ} \mathrm{C} ; 95-97 \%$ UR) e $\mathrm{AC}$, mais quatro dias em condição ambiente $\left(20 \pm 2^{\circ} \mathrm{C} ; 60 \pm 5 \%\right.$ UR).

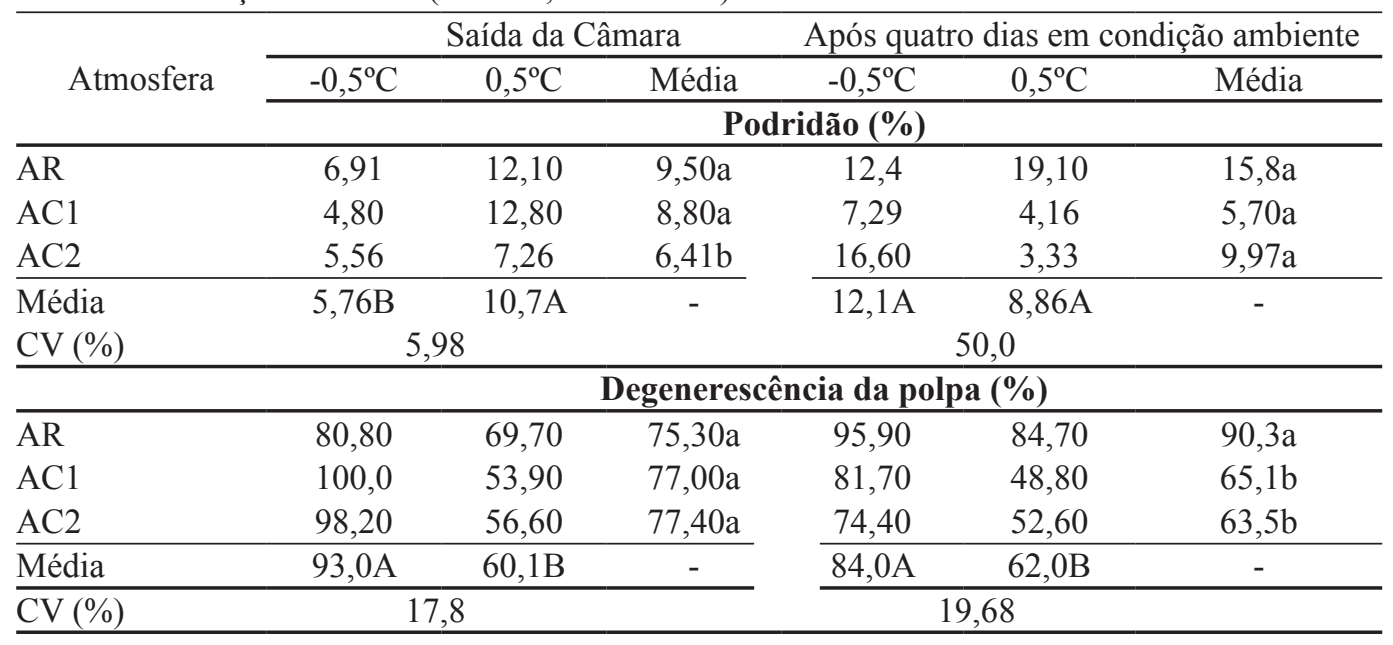

Médias seguidas por mesma letra, maiúscula na horizontal, entre temperaturas (para uma mesma data de avaliação), e minúscula na vertical, entre atmosferas de armazenamento, não diferem entre si, pelo teste de Tukey $(\mathrm{p}<0,05)$. $\mathrm{AR}\left(21 \mathrm{kPa} \mathrm{O}_{2}+<0,03 \mathrm{kPa} \mathrm{CO}_{2}\right)$, $\mathrm{AC} 1\left(1 \mathrm{kPaO}_{2}+3 \mathrm{kPaCO}\right)$ e $\mathrm{AC} 2\left(2 \mathrm{kPaO}_{2}+5 \mathrm{kPaCO}_{2}\right)$

\section{CONCLUSÃO}

O armazenamento em AC retarda o amadurecimento dos frutos, com a melhor condição envolvendo o emprego de $2,0 \mathrm{kPa}$ de $\mathrm{O}_{2}+5,0 \mathrm{kPa}$ de $\mathrm{CO}_{2}$, na temperatura de $0,5^{\circ} \mathrm{C}$. No entanto, o armazenamento da ameixa 'Laetitia', nas condições de AC avaliadas, não pode estender-se por 60 dias, já que resulta em elevada degenerescência da polpa.

\section{AGRADECIMENTOS}

Os autores agradecem ao Conselho Nacional de Desenvolvimento Científico e Tecnológico (CNPq), à Fundação de Amparo à Pesquisa e Desenvolvimento Científico e Tecnológico de Santa Catarina (FAPESC) e à Coordenação de Aperfeiçoamento de Pessoal de Nível Superior (CAPES), pelo apoio financeiro a este projeto. 


\section{REFERÊNCIAS}

ARGENTA, L.C.; KRAMMES, J.G.; MEGGUER, C.A.; AMARANTE, C.V.T.; MATTHEIS, J. Ripening and quality of 'Laetitia' plums following harvest and cold storage as affected by inhibition of ethylene action. Pesquisa Agropecuária Brasileira, Brasília, v.38, n.10, p.139-1148, 2003.

BLANKENSHIP, S.M.; DOLE, J.M. 1-Methylcyclopropene: a review. Postharvest Biology and Technology, Amsterdam, v.28, p.1-25, 2003.

BRACKMANN, A.; BORDIGNON, B.C.S.; GIEHL, R.F.H.; SESTARI, I.; EISERMANN, A.C. Armazenamento de pêssegos cv. "Granada" em atmosfera controlada, visando ao transporte a longas distâncias. Ciência Rural, Santa Maria, v.37, n.3, p.676-681, 2007.

BRACKMANN, A.; STEFFENS, C.A.; GIEHL, R.F.H. Armazenamento de pêssegos 'Chimarrita' em atmosfera controlada e sob absorção de etileno. Ciência Rural, Santa Maria, v.33, n.3, p.431-435, 2003.

BRACKMANN, A.; STEFFENS, C.A.; MELLO, A.M.; VIZZOTTO, M. Armazenamento de ameixas 'Reubennel' e 'Pluma 7' e frigoconservação intermitente e atmosfera controlada. Revista Científica Rural, Bagé, v.6, n.1, p.71-76, 2001.

BRACKMANN, A.; WEBER, A.; PINTO, J.A.V.; NEUWALD, D.A.; STEFFENS, C.A. Manutenção da qualidade pós-colheita de maçãs 'Royal Gala' e 'Galaxy' sob armazenamento em atmosfera controlada. Ciência Rural, Santa Maria, v.38, n.9, p.24782484, 2008

DRAKE, S.R.; ELFVING, E. Short-term controlled atmosphere storage for storage-life extension of white-fleshed peaches and nectarines. Journal of Food Quality, Amsterdam v.26, p.135-147, 2003.

FONSECA, S.C.; OLIVEIRA, F.A.R.; BRECHT, J.K. Modelling respiration rate of fresh fruits and vegetables for modified atmosphere packages: a review. Journal of Food Engineering, Amsterdam, v.52, p.99-119, 2002.
GIRARDI, C.L.; CURRENT, A.R.; LUCCHETTA, L.; ZANUZO, M.R.; DA COSTA, T.S.; BRACKMANN, A.; TWYMAN, R.M.; NORA, F.R.; NORA, L.; SILVA, J.A.; ROMBALDI, C.V. Effect of ethylene, intermittent warming and controlled atmosphere on postharvest quality and the occurrence of woolliness in peach (Prunus persica cv. Chiripá) during cold storage. Postharvest Biology and Technology, Amsterdam, v.38, n.1, p.25-33, 2005.

GORNY, J.R.; KADER, A.A. The mode of $\mathrm{CO}_{2}$ action on ACC oxidase and its role in inhibition of ethylene biosynthesis. HortScience, Alexandria, v.29, n.5, p.533, 1994

JAYAS, D.S.; JEYAMKONDAN, S. Modified atmosphere storage of grains, meats, fruits and vegetables. Biosystems Engineering, Amsterdam, v.82, n.3, p.235-251, 2002.

KADER, A.A. Respiration and gas exchange of vegetables. In: WEICHMANN, J. (Ed.). Postharvest physiology of vegetables. New York: M. Dekker, 1987. p.25-43.

LIU, S.; YANG, Y.; MURAYAMA, H.; TAIRA, S.; FUKUSHIMA, T. Effects of $\mathrm{CO} 2$ on respiratory metabolism in ripening banana fruit. Postharvest Biology and Technology, Amsterdam, v.33, p.2734, 2004.

MAJUMDER, K.; MAZUMDAR, B.C. Changes of pectic substances in developing fruits of capegooseberry (Physalis peruviana L.) in relation to the enzyme activity and evolution of ethylene. Scientia Horticulturae, Amsterdam, v.96, n.1-4, p.91-101, 2002.

MANGANARIS, G.A.; VICENTE, A.R.; CRISOSTO, C.H.; LABAVITCH, J.M. Cell wall modifications in chilling-injured plum fruit (Prunus salicina). Postharvest Biology and Technology, Amsterdam, v.48, p.77-83, 2008.

MATHOOKO, F.M. Regulation of respiratory metabolism in fruits and vegetables by carbon dioxide. Postharvest Biology and Technology, Amsterdam, v.9, p.247-264, 1996. 
MURRAY, R. ; LUCANGELI, G.; POLENTS, C.; BUDDE, C. Combined pre-storage heat treatment and controlled atmosphere storage reduced internal breakdown of 'Flavorcrest' peach. Postharvest Biology and Technology, Amsterdam ,v.44, p.116121, 2007.

SAQUET, A.A.; BRACKMANN, A.; STORCK, L. Armazenamento de maçã 'Gala' sob diferentes temperaturas e concentrações de oxigênio e gás carônico. Ciência Rural, Santa Maria, v.27, n.3, p.399-405, 1997.

SESTARI, I.; GIEHL, R.F.H.; PINTO, J.A.V.; BRACKMANN, A. Condições de atmosfera controlada para pêssego "Maciel" colhido em dois estádios de maturação. Ciência Rural, Santa Maria, v.38, n.5, p.1240-1245, 2008.

STEFFENS, C.A.; BRACKMANN, A.; PINTO, J.A.V.; EISERMANN, A.C. Escurecimento da polpa e respiração de pêssegos em função das condições de armazenamento. Revista Brasileira Agrociências, Pelotas, v.12, n.1, p.71-75, 2006.
STEFFENS, C.A.; BRACKMANN, A.; PINTO, J.A.V.; EISERMANN, A.C. Taxa respiratória de frutas de clima temperado. Pesquisa Agropecuária Brasileira, Brasília, v.42, n.3, p.313-321, 2007.

STRIEF, J. Lagerung von Stein und Beerebobst. Besseres Obst, Osterreich, v.4, p.18-19, 1995.

WILLS, R.H.H.; LEE, T.H.; GRAHAM, D.; McGLASSON, W.B.; HALL, E.G. Postharvest: an introduction to the physiology and handling of fruit, vegetables and ornamentals. $4^{\text {th }}$ ed. New York: $C A B$ International, 1998. 262p.

ZHOU, H.W.; LURIE, S.; LERS, A.; KHATCHITSKI, A.; SONEGO, L.; BEN-ARIE, R. Delayed storage and controlled atmosphere storage of nectarines: two strategies to prevent woolliness. Postharvest Biology and Technology, Amsterdam, v.18, p.133$141,2000$. 